\title{
Study on the Turbulent Flow of Ag-Ga Nanofluid in a Spirally Corrugated Tube
}

\author{
Cong Qi \& Chunyang Li* \& Chengyuan Luo \\ School of Electric Power Engineering, China University of Mining and Technology, Xuzhou 221116, China
}

*Corresponding author: Chunyang Li

\begin{abstract}
In order to study the turbulent flow characteristics of nanofluid, this paper establish the nanofluid turbulent flow model in a spirally corrugated tube. In the condition of a certain temperature difference between import and export $(\triangle T=60 \mathrm{~K})$, different concentrations of nanoparticles $(\varphi=0.01, \varphi=0.03$ and $\varphi=0.05$ ) of Ag-Ga nanofluid turbulent flow characteristics is simulated in the spirally corrugated tube. Considering the change of temperature, pressure, velocity and so on in the spiral corrugated tube. Resistance loss is studied under different concentrations of $\mathrm{Ag}$-Ga nanofluid in spirally corrugated tube. The research results show that compared with smooth tube, tube resistance loss coefficient of Ag-Ga nanofluid in the spiral corrugated tube resistance loss coefficient is larger, and it enlarge with the increasing of nano silver particles size.

KEYWORD: liquid metal base nanofluid; turbulent flow; spirally corrugated tube; resistance loss
\end{abstract}

\section{INTRODUCTION}

Since the $1970 \mathrm{~s}$, the energy crisis appears. Experts and scholars must accelerate the study of new type of high efficient heat transfer working medium to deal with the challenges, and research how to strengthen heat transfer performance (A. Koca et al, 2007). With the rapid development of computer technology, methods to simulate the heat transfer effect of the nanofluid with the use of software attract the attention of people (M. Sheikholeslami et al, 2014) . Maiga and Gines (S.E.B. Maiga et al, 2005) (R. Gilles et al, 2004) used FLUENT software to analog compute the influence of components of the solid nano dispersed phase to forced convection heat transfer at corresponding working medium in $\mathrm{Al}_{2} \mathrm{O}_{3}-$ $\mathrm{H}_{2} \mathrm{O}$ and $\mathrm{Al}_{2} \mathrm{O}_{3}$-ethylene glycol nanofluid. Heris etc. (M.M. Rashidi et al, 2014) also used FLUENT to study the influence of components of $\mathrm{CuO}$ and $\mathrm{Al}_{2} \mathrm{O}_{3}$ nano-particles to corresponding water-based nanofluid heat transfer performance.

Nevertheless, when refer to the metal-based nanofluid turbulent flow numerical simulation, there is few research results at present. This paper takes gallium metal base fluid and silver nanoparticles as study object, to research its pressure loss in the spiral grooved tube.

\section{METHODS}

\subsection{Physical model.}

This paper adopted the nanofluid with concentrations of $0.01,0.03,0.05$, whose physical parameters are shown in Table 1.

This paper established the spiral fluted tube model (Fig.1).Tubeline is $L_{0}=200 \mathrm{~mm}$ long, whose spiral fluted tube is $L=100 \mathrm{~mm}$ long; Tube diameter is D $=20 \mathrm{~mm}$ wide; Spiral groove pitch is $\mathrm{T}=\mathrm{D} / 4=5$ $\mathrm{mm}$; Spiral groove depth and width are $\mathrm{a}=\mathrm{D} / 10=$ $2 \mathrm{~mm}$. Silver- gallium nanofluid (Ag - Ga) was taken as a heat transfer medium to research the influence of different composition of silver particles on the flow.

Table $1 \mathrm{Ag}$ - Ga nanofluid physical property parameter

\begin{tabular}{cccccc}
\hline $\begin{array}{c}\text { volume } \\
\text { fraction }(\varphi)\end{array}$ & $\begin{array}{c}\text { density } \\
\left(\mathrm{kg} / \mathrm{m}^{3}\right)\end{array}$ & specific heat $(\mathrm{J} / \mathrm{kg} \cdot \mathrm{K})$ & $\begin{array}{c}\text { Thermal conductivity } \\
\left(\mathrm{W} / \mathrm{m}^{2} \cdot \mathrm{K}\right)\end{array}$ & $\begin{array}{c}\text { dynamic viscosity } \\
(\mathrm{kg} / \mathrm{m} \cdot \mathrm{s})\end{array}$ & $\begin{array}{c}\text { Coefficient of thermal } \\
\text { expansion }\left(\mathrm{K}^{-1}\right)\end{array}$ \\
\hline 0.01 & 6134 & $429.9-0.275543 \mathrm{~T}$ & 31.6422 & 0.001935936 & 0.00009803 \\
0.03 & 6222 & $429.9-0.275543 \mathrm{~T}$ & 32.9536 & 0.002037275 & 0.00009409 \\
0.05 & 6310 & $429.9-0.275543 \mathrm{~T}$ & 34.3023 & 0.002146199 & 0.00009015 \\
\hline
\end{tabular}




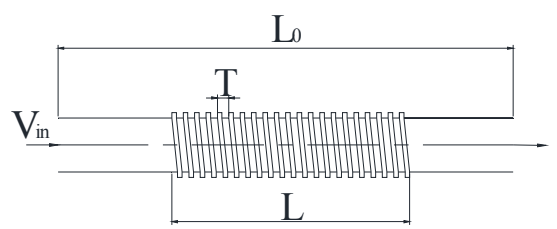

Fig.1 Spirally corrugated tube schematic diagram

\subsection{Mathematical model.}

The magnitude of silver nanoparticles in nanofluid are nanoscale, which makes the properties of the nanoparticles are similar to the characteristics of metal base extremely. It means that nanofluid can be treated as a single phase fluid. Liquid metal based nanofluid flow is affected by the control equation. The basic control equations are as follows (Y. He et al, 2009).

Continuity equation

$\frac{\partial \rho_{l}}{\partial t}+\nabla \cdot\left(\rho_{l} \mathbf{v}_{l}\right)=0$

\section{Momentum equation}

$\frac{\partial \rho_{l} \mathbf{v}_{l}}{\partial t}+\nabla \cdot\left(\rho_{l} \mathbf{v}_{l} \mathbf{v}_{l}\right)=-\nabla p+\nabla \cdot \mathbf{T}_{l}+\rho_{l} \mathbf{g}$

Among them, $p$ is a fluid pressure, stress tensor $T_{l}$ are defined as follows:

$\mathbf{T}_{l}=\mu_{l}\left[\nabla \mathbf{v}_{l}+\nabla \mathbf{v}_{l}{ }^{T}\right]-\frac{2}{3} \mu_{l} \nabla \cdot \mathbf{v}_{l} \mathbf{I}$

Among them, $\mu_{l}$ is the dynamic viscosity of liquid metal fluid viscosity, $I$ is the unit tensor.

Energy equation

$\rho_{l} c\left[\frac{\partial T}{\partial t}+v_{l} \cdot \nabla T\right]=\nabla \cdot[k \nabla T]$

Among them, $c$ is the specific heat of liquid metal fluid. $k_{l}$ is the thermal conductivity of liquid metal fluid and $T$ is the temperature of the liquid metal fluid.

The flow of the spiral fluted section pressure loss:

$\Delta p=p_{-5}-p_{105}$

Among them $p_{-5}$ is the pressure of $\mathrm{x}=5$ in the tube, and $p_{-105}$ is the pressure of $\mathrm{x}=105$.

Loss coefficient:

$\lambda=\frac{2 \Delta p}{\rho V^{2}} \frac{D}{L}$

\section{RESULTS AND DISCUSSIONS}

In this paper, Ag - Ga nanofluids in 0.01, 0.03, and 0.05 components were simulated, and get a series of cloud image.
In order to study the nanofluid heat transfer characteristics, temperature distribution cloud images of different components nanofluid in the tube are made (Fig 2).It shows that, with the increase of the composition of nano fluid, in the high temperature area at tube from high temperature to low temperature, temperature change range narrow and the thinning of the laminar boundary layer from figure 3, which means that heat transfer accelerate. Nanoparticles sped up the heat transfer rate, and improve the coefficient of thermal conductivity.

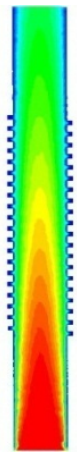

(a)

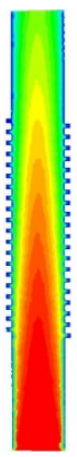

(b)

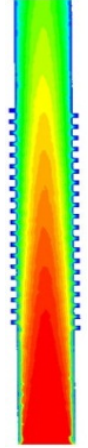

(c)

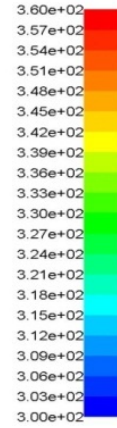

$3.000+0$
Fig.2 Nephogram of temperature for different components of nanofluid

(a) $0.01 ;$ (b) 0.03 ; (c) 0.05

In order to study the speed of change in the tube, the velocity distribution cloud images of different components nanofluid were made (Fig.3). It can be seen from the Fig. 3 that the velocity of 0.01 nanoflu$\mathrm{id}$ is the fastest, and the velocity of 0.05 nanofluid is the lowest. Nanoparticle influenced the movement of the nanofluid in the tube, which makes friction force increases, speed decreases, and flow velocity slows down. Velocity is larger in tube center, and edge speed is smaller.

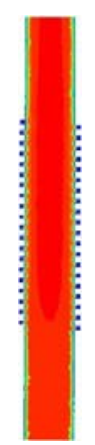

(a)

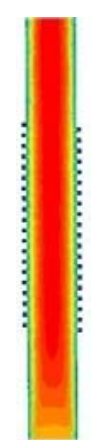

(b)

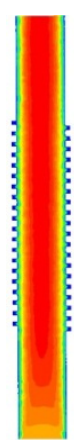

(c)

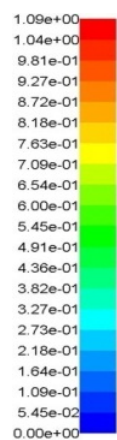

$5.45 e-02$
Fig.3 Nephogram of velocity for different components of nanofluid (a) $0.01 ;$ (b) 0.03 ; (c) 0.05

In order to study the stress change of nanofluid in the tube, pressure distribution cloud images of different components of nanofluid were made (Fig.4). It can be seen from the Fig.4 that nanofluid inlet pressure is larger in the tube, and outlet pressure is 
smaller to zero. Nanofluid pressure change rate is faster in thread area, but nanofluid pressure rate change is slower in excluding thread area. The bigger composition of nanofluid is, more quickly the pressure change.

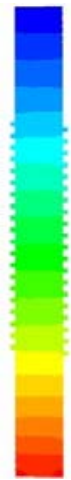

(a)

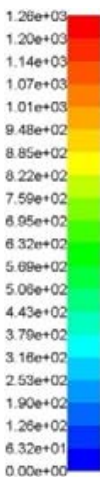

(b)
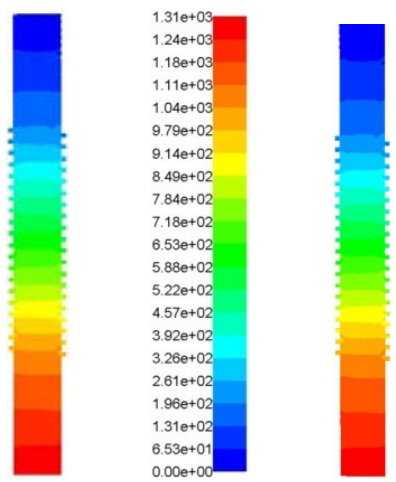

(c)

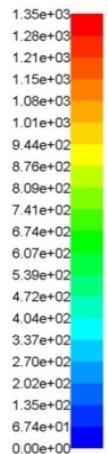

Fig.4 Nephogram of pressure for different components of nanofluid

(a) 0.01 ; (b) 0.03 ; (c) 0.05

\section{CONCLUSION}

This paper established the Ag-Ga nanofluid turbulent flow model, and simulated Ag-Ga nanofluid turbulent flow on the smooth tube and spiral grooved tube respectively. The conclusions are as following:

(1) With the increase of nanofluid composition, physical characteristics of nanofluid in spiral grooved tube are obviously different, which makes heat accelerated, speed decreased, pressure transformation was accelerated.

(2) Compared with smooth tube, Ag-Ga nanofluid in the spiral grooved tube resistance loss increased.

(3) As component of silver nanoparticles in Ag $\mathrm{Ga}$ fluid increases, the resistance loss increases gradually, and the biggest difference appears at the $R e=25000$.

(4) As Re increases, the spiral fluted tube resistance loss coefficient is on the decline.

\section{ACKNOWLEDGEMENTS}

This work is financially supported by the China Postdoctoral Science Foundation funded project (NO. 2014M551694).

\section{REFERENCES}

A. Koca, H.F. Oztop and Y. Varol: International Communications in Heat and Mass Transfer, Vol.34 (2007), No.4, p. 511-519.
M.M. Rashidi, N. Kavyani and S. Abelman: International Journal of Heat and Mass Transfer, Vol 70(2014), p. 892917.

M. Sheikholeslami, M.G. Bandpy, R. Ellahi and et al: Journal of Magnetism and Magnetic Materials, Vol. 369(2014), p. 69-80.

R. Gilles, C.T. Nguyen and P.R. Lajoie: Superlattices and Microstruetures, Vol. 35(2004), p. 497-511.

S.E.B. Maiga, S.J. Palm, C.T. Nguyen: International Journal of Heat and Fluid Flow, Vol 26(2005), p. 530-546.

Y. He, Y. Men, Y. Zhao, H. Lu, Y. Ding: Applied Thermal Engineering, Vol 29(2009), No.10, p. 1965-1972. 